\title{
Multi-Frequency Interference Detection and Mitigation Using Multiple Adaptive IIR Notch Filter with Lattice Structure
}

\author{
Abdelrahman El Gebali, René Jr Landry \\ Lassena Laboratory, Department of Electrical Engineering, École de Technologie Supérieure (ÉTS), Montreal, Canada \\ Email: abdelrahman.elgebali@lassena.etsmtl.ca
}

How to cite this paper: El Gebali, A. and Landry, R.J. (2021) Multi-Frequency Interference Detection and Mitigation Using Multiple Adaptive IIR Notch Filter with Lattice Structure. Journal of Computer and Communications, 9, 58-77.

https://doi.org/10.4236/jcc.2021.95005

Received: April 20, 2021

Accepted: May 17, 2021

Published: May 20, 2021

Copyright $\odot 2021$ by author(s) and Scientific Research Publishing Inc. This work is licensed under the Creative Commons Attribution International License (CC BY 4.0).

http://creativecommons.org/licenses/by/4.0/

\begin{abstract}
Radio Frequency Interferences (RFI), such as strong Continuous Wave Interferences (CWI), can influence the Quality of Service (QoS) of communications, increasing the Bit Error Rate (BER) and decreasing the Signal-to-Noise Ratio (SNR) in any wireless transmission, including in a Digital Video Broadcasting (DVB-S2) receiver. Therefore, this paper presents an algorithm for detecting and mitigating a Multi-tone Continuous Wave Interference (MCWI) using a Multiple Adaptive Notch Filter (MANF), based on the lattice form structure. The Adaptive Notch Filter (ANF) is constructed using the secondorder IIR NF. The approach consists in developing a robust low-complexity algorithm for removing unknown MCWI. The MANF model is a multistage model, with each stage consisting of two ANFs: the adaptive IIR notch filter $H_{l}(z)$ and the adaptive IIR notch filter $H_{N}(z)$, which can detect and mitigate CWI. In this model, the ANF $H_{l}(z)$ is used for estimating the Jamming-to-Signal Ratio (JSR) and the frequency of the interference $\left(\omega_{0}\right)$ by using an LMS-based algorithm. The depth of the notch is then adjusted based on the estimation of the JSR. In contrast, the ANF $H_{N}(z)$ is used to mitigate the CW interference. Simulation results show that the proposed ANF is an effective method for eliminating/reducing the effects of MCWI, and yields better system performance than full suppression $\left(k_{N}=1\right)$ for low JSR values, and mostly the same performance for high JSR values. Moreover, the proposed can detect low and high JSR and track hopping frequency interference and provides better Bit error ratio (BER) performance compared to the case without an IIR notch filter.
\end{abstract}

\section{Keywords}

Component, Radio Frequency Interference (RFI), Multiple Adaptive Notch Filter (MANF), Jamming-to-Signal Ratio (JSR), Quality of Service (QoS), BER 


\section{Introduction}

Many communications systems, such as wireless and satellite communication systems, suffer from Radio Frequency Interference (RFI) thanks to the ever-increasing number of wireless applications in use, as well as human-made RFI. RFI may be unintentional or could be an intentional act, such as jamming. It can also reduce the Signal of Interest (SoI) and result in a degraded QoS, a decrease in SNR, and an increase in BER or a loss of the receiver's communication signal. Various jamming mitigation techniques have been proposed in the literature, and can be classified as adaptive antenna-based techniques (space domain) [1], Time-Frequency Filtering (TFF)-based methods [2] [3], and adaptive filtering-based techniques [4] [5] [6] [7]. The adaptive antenna methods (spatial filtering) are appropriate for Narrow-Band (NB) and wideband (WB) jamming mitigations. The space domain (antenna array) can also achieve a high jammer suppression ratio but is not suitable for mobile devices [8] [9]. The TFF methods remove signal features in both the Time Domain (TD) and the Frequency Domain (FD) simultaneously, but the major concern with the FD method is computational complexity, which arises from FFT and IFFT in hardware. [10]. The methods are appropriate for NB jamming mitigation, such as Short Time Fourier transforms (STFT) [11], bilinear signal distribution [12], filter banks [13], and Wavelet Transforms (WT) [14] [15]. The STFT and the filter bank techniques employ fixed windows, which are useful for analyzing nonstationary signals. The adaptive filtering-based methods are commonly used for narrowband CWI jamming rejection and can be classified into two approaches, namely, TD and FD. For the FD approach, [16] proposed an N-sigma method to reject CWI. Also, [17] [18] proposed methods to detect and mitigate CWI and NB interference. Also, [19] proposed a method to detect and mitigate MCWI using a first-order IIR notch filter based on a simplified Welch algorithm and notch filter. However, the main concerns with the FD approach are computational complexity and higher costs. Using the Finite Impulse Response (FIR) and the Infinite Impulse Response (IIR) filters to detect and mitigate interference for TD approaches. The papers by [20] [21] [22] present methods for suppressing the CWI in the TD using an ANF based on a lattice form structure. The authors in [23] proposed a method for detecting and mitigating $\mathrm{CW}$ interference using two-pole and multiple NF. Also, [9] presented a technique using ANF to detect and mitigate MCWI in GPS. In [10], the ANF is used to mitigate both CWI and NB interference for GNSS. Moreover, the papers by [24] [25] [26] present linear prediction and nonlinear prediction methods. Both methods require longer tap filters to effectively mitigate narrowband interference, which increases the system's complexity [20]. In the literature, NF is used to reduce and remove interference in different applications, such as biomedical applications and [27] and GNSS applications (e.g., DSSS systems and GPS receivers) [20] [23]. The lattice IIR ANF performs better than the direct IIR ANF form [21] [22]. The IIR NF in lattice form is less computationally complex than are direct form implementations [20] [21] [23]. 
A typical IIR notch digital filter has constrained zeros on the unit circle, which makes the notch more depth "results into infinite" leading to complete removal of the interference. Nevertheless, this method creates self-noise due to the distortion of the data "desired signals" at the notch frequency as the interference is removed [22], called self-noise, as briefly described in [28]. In DSSS systems, Choi [20] proposed a new CWI excision method that uses an ANF to reduce self-noise. The authors in [29] proposed a novel low-complexity anti-jamming filter to reduce the CWI for general wireless communications. With the exception of the works in [20] and [29], these previous works concentrated on detecting and removing CWI and MCWI without focusing on controlling the notch filter's depth. The two divergent papers [20] [29] focused only on the excision of the CWI. The present paper proposes a low-complexity algorithm operating in TD using multiple ANF to detect and mitigate MCWI with considering controlling the notch depth based on an estimation of the interference power, called an anti-jamming receiver system model. This model is constructed using the secondorder IIR NF based on a lattice form structure described in Section 3.

The rest of the paper is organized as follows. The signal model of the transmitted and received signals in the presence of MCWI is described in Section 2. Section 3 reviews and describes the adaptive IIR NF structure and the proposed adaptation algorithm for mitigating MCWI using MANF. Section 4 describes and defines the optimal notch depth that maximizes the output SNR, while Section 5 presents the simulation performed and discusses the results obtained. Finally, Section 6 concludes the paper.

\section{Signal Model}

In this section, Figure 1 presents the transmitted and received signal model, where a Quadrature Phase Shift Keying (QPSK) signal and an interference signal with the AWGN are generated using a MATLAB simulation model.

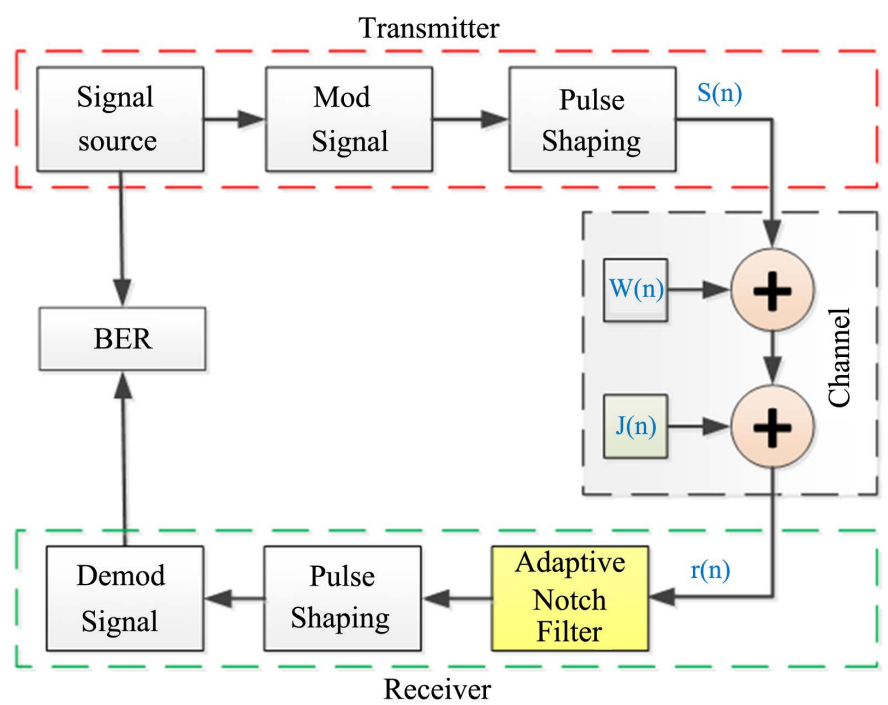

Figure 1. Signal model of the transmitted and received signals block diagram. 


\subsection{Interference Signal Model}

Continuous Wave Interference (CWI) is one of the most common sources of interference. CWI can be modeled as a sinusoidal wave in time, such as a single or multi-CWI. A multitoned CWIs is considered in this paper, and can be modeled as:

$$
J_{i}(t)=\sum_{i=1}^{m} A_{i} \cos \left(2 \pi f_{i} t+\theta_{i}\right)
$$

where:

- $m$ is the number of CW interferences;

- $A_{i}$ is the amplitude of the $i^{\text {th }}$ interference signal;

- $f_{i}$ is the frequency of the $i^{\text {th }}$ interference signal;

- $\theta_{i}$ is the phase delay of the $i^{\text {th }}$ interference signal, which is assumed to be a random variable uniformly distributed in the range $[-\pi, \pi]$.

\subsection{Received Signal Model}

The received signal $r(t)$, which is the input signal to the IIR notch filter, is the sum of the QPSK modulated baseband signal $S(t)$, the AWGN, $w(t)$, and the interference signal, $J_{i}(t)$, which can be modeled as:

$$
r(t)=S(t)+w(t)+J_{i}(t)
$$

In Section 3, the case of a multitoned CWI is considered, and $m=2$. The input baseband signal to the IIR notch filter $r(t)$ given by Equation (2) is then sampled at chip rate to convert it into discrete-time samples for further processing as shown here:

$$
r\left(n T_{s}\right)=S\left(n T_{s}\right)+w\left(n T_{s}\right)+\sum_{i=1}^{m} A_{i} \cos \left(2 \pi f_{i} n T_{s}+\theta_{i}\right)
$$

For ease of notation, the sampling interval $T_{s}$ will be ignored in the rest of this paper, and Equation (3) will be adopted as:

$$
r(n)=S(n)+w(n)+\sum_{i=1}^{m} A_{i} \cos \left(2 \pi f_{i} n+\theta_{i}\right)
$$

where:

- $S(n)$ is the desired QPSK modulated signal;

- $w(n)$ is the AWGN.

\section{Proposed Algorithm and Structure of the Filter}

This paper proposes using a MANF based on a second-order IIR lattice form structure as an anti-jamming receiver system model, as shown in Figure 2. The model presents as a low-complexity algorithm operating in the TD approach. The reason the approach operates in TD is to avoid the costs associated with the transfer domain approach. The MANF system model is a multistage model, with each of its stages consisting of two ANFs: the adaptive IIR notch filter $H_{l}(z)$ and the adaptive IIR notch filter $H_{N}(z)$. The transfer functions of the two ANFs $\left[H_{l}(z)\right.$ and $\left.H_{N}(z)\right]$ are described in Section 3.1. The ANF $H_{l}(z)$ transfer function has zeros on the unit circle, resulting in the notch depth going 


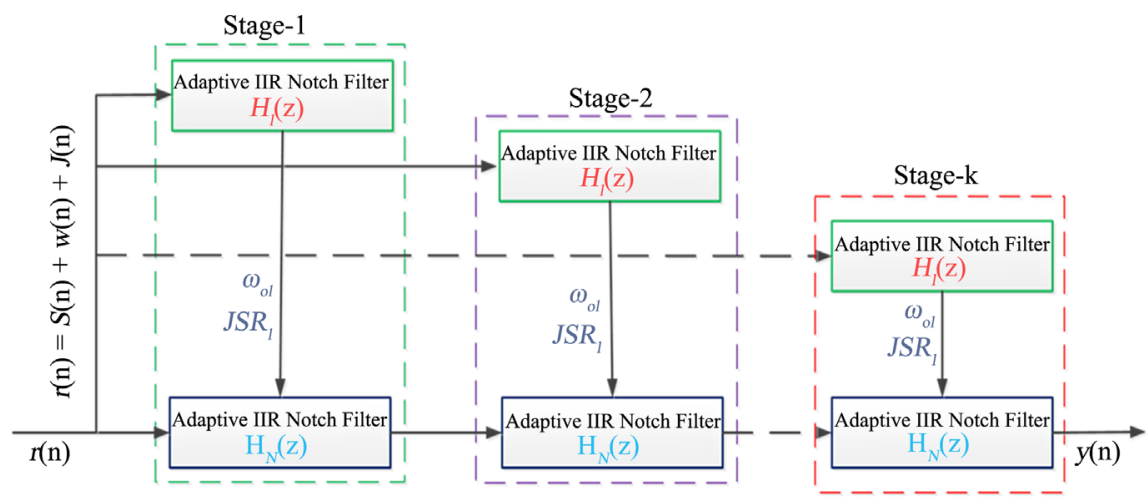

Figure 2. An anti-jamming receiver system model of MANF block diagram.

into infinite, and leading to the complete removal of the interference and exclusion of some desired signals. To avoid this, the ANF $H_{N}(z)$ is added to adjust and control the depth of the notch $\left(k_{N}\right)$ according to the estimated $\mathrm{JSR}_{l}$ in order to reduce the elimination of some useful signals while removing interferences. The adaptive filter can be performed in various structures or realizations. There are two major implementations of an adaptive digital notch filter: the FIR and the IIR. FIR filters are also called moving average (MA) filters, which implement an all-zero transfer function. While the IIR filter has two parts, the auto-regression (AR) and the moving average (MA) (or an ARMA), the filter implements all-pole and all-zero transfer functions. In this work, the IIR filter is used since the IIR NF obtains a frequency response closer to that of the ideal NF than do FIR notch filters of the same length. Figure 3 describes the adaptive IIR notch filter algorithm structure $H_{l}(z)$ [21] that attempts to adjust the $k_{o l}$ of the notch filter, which consists of two parts, the AR block and the MA block. The MA block is responsible for removing the interference signal, but it also removes the signal near the interference frequency. The ANF $H_{l}(z)$ is used to detect and estimate $\mathrm{JSR}_{l}$ and the frequency of the interference $\left(\omega_{o l}\right)$, while the $\operatorname{ANF}\left(H_{N}(z)\right)$ is used for interference cancellation. Note that in this work, the number of states is chosen as two $(k=2)$.

Figure 4 introduces the multiple adaptive IIR NF implementation, similar to the cascade filter, but with an individual adaptive algorithm. The multiple adaptive IIR NF is implemented by having two different notch frequencies.

\subsection{Transfer Function of IIR Notch Filter}

This section introduces the second-order IIR NF transfer function $H_{l}(z)$ based on the lattice form structure. A decision was made to use the IIR NF with an LMS algorithm to design and implement the Adaptive Notch Filter (ANF). By indicating the adaptive coefficient of $I^{t h}$ ANF by $k_{o l}$, the second-order IIR NF transfer function $H_{l}(z)$ [16] is defined as:

$$
H_{l}(z)=\frac{N_{l}(z)}{D_{l}(Z)}=\frac{1+2 k_{o l} Z^{-1}+Z^{-2}}{1+k_{o l}(1+\beta) Z^{-1}+\beta Z^{-2}}
$$




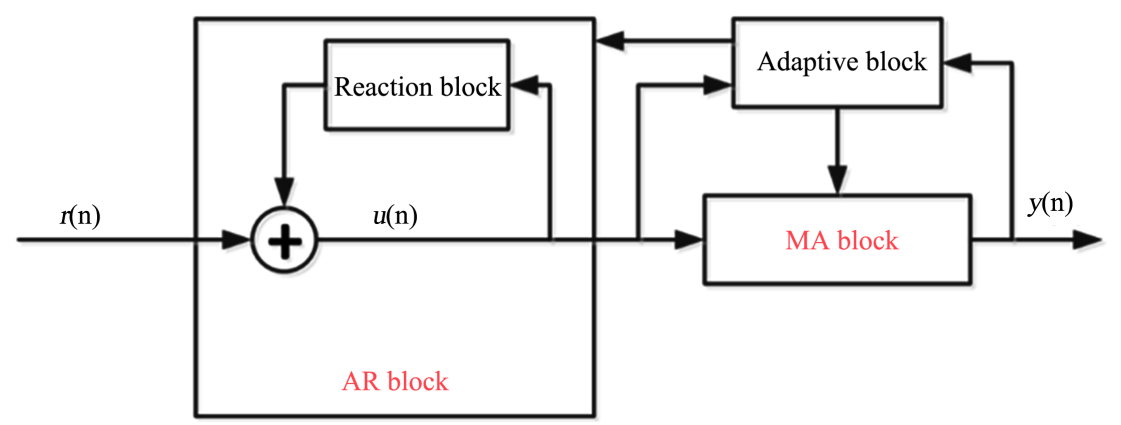

Figure 3. Structure of ANF $H_{l}(z)$.

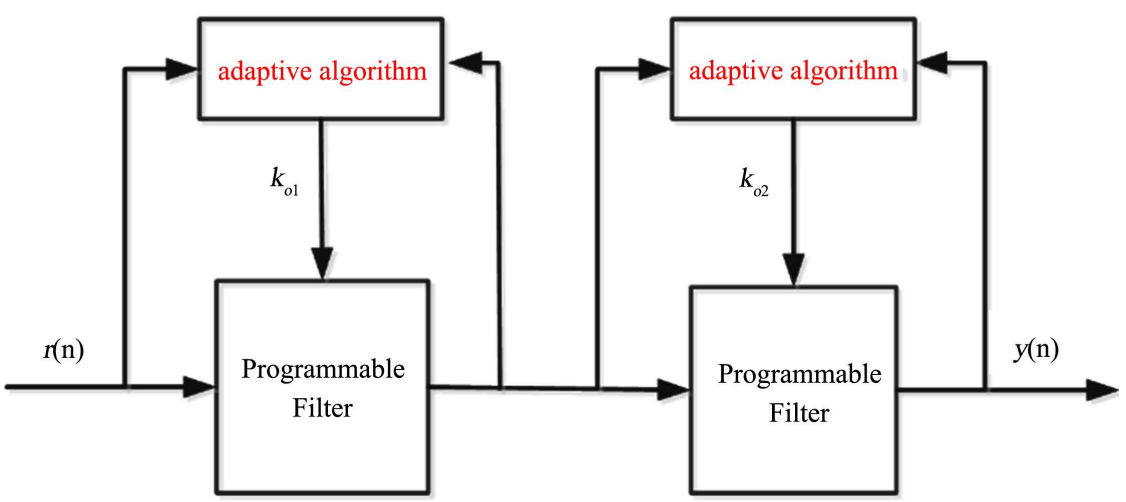

Figure 4. Multiple adaptive IIR notch filter.

where $\beta$ is the pole radius factor that controls the filter's bandwidth $(0<\beta<1)$; $k_{o l}$ is a coefficient of lattice IIR NF parameter that rejects an unknown CWI, which is defined as $k_{o l}=-\cos \left(\omega_{o l}\right)$. If the absolute values of both $\beta$ and $k_{o l}$ are less than 1 , the $H_{l}(z)$ is stable. Also, in this work, the parameter $\beta$ is fixed and only adapt $k_{o l}$ to avoid notch too much QPSK signal.

\subsection{Adaptation of Parameter $\boldsymbol{k}_{o l}$ and LMS Adaptive Algorithm}

In this subsection, the derivation of the LMS adaptive algorithm is utilized in transversal tapped (IIR) filters with the structure shown in Figure 3. It is assumed that the MCWI and the AWGN are added to the QPSK signal described in Equation (4). $y(n)$ and $u(n)$ are the outputs of the MA and the AR block, respectively, as shown in Figure 3, which can be defined in terms of the signal that was received $r(n)$ as given by:

$$
\begin{gathered}
y(n)=u(n)+2 k_{o l} u(n-1)+u(n-2) \\
u(n)=r(n)-k_{o l}(1+\beta) u(n-1)-\beta u(n-2)
\end{gathered}
$$

The cost function of the filter can be given as [30]:

$$
J\left(k_{o l}\right)=E\left\{|y(n)|^{2}\right\}
$$

where $E\{*\}$ is an expectation operator. Replacing the expectation value in Equation (8) with the ensemble-averaged value as shown in Equation (9), we get: 


$$
\dot{J}\left(k_{o l}\right)=\frac{1}{L} \sum_{n=0}^{L-1} y^{2}(n)
$$

where $L$ is the length of data. The goal of the filter is to adjust the notch parameter $k_{o}$ for minimizing the cost function, and the gradient-based method of steepest descent is used to achieve the goal:

$$
k_{o l}(n+1)=k_{o l}(n)-\mu\left(\frac{\mathrm{d} \dot{J}\left(k_{o l}\right)}{\mathrm{d} k_{o l}}(n)\right)
$$

The instantaneous value of $\dot{J}\left(k_{o}\right)$ is used to replace the ensemble-averaged value as:

$$
k_{o l}(n+1)=k_{o l}(n)-\mu\left(\frac{\mathrm{d} y^{2}(n)}{\mathrm{d} k_{o l}(n)}\right)
$$

To simplify, Equation (11) thus becomes:

$$
k_{o l}(n+1)=k_{o l}(n)-\mu y(n)\left(g\left(J\left(k_{o l}\right)\right)\right)
$$

where $g\left(J\left(k_{o l}\right)\right)$ is the gradient signal for the adaptation of $k_{o l}$, and is defined as:

$$
g\left(J\left(k_{o l}\right)\right)=-(1+\beta) \times(n-1)
$$

Substituting into Equation (13):

$$
k_{o l}(n+1)=k_{o l}(n)-\mu y(n)((1-\beta) x(n-1))
$$

where $\mu$ is the step-size factor that controls the convergence speed and stability, and Equation (14) is a new adaptive algorithm for our proposed ANF that allows and controls the update filter coefficient in this work.

Since the normalized notch frequencies, $f_{N}$ and $k_{o l}$, are related by $k_{o l}=-\cos \left(\omega_{o l}\right)$, where $\omega_{o l}=2 \pi f_{N}$, at sample $n$, the estimated frequency is given by:

$$
\hat{f}_{N}(n)=\frac{1}{2 \pi} \arccos \left(\hat{k}_{o l}(n)\right)
$$

The estimated $\mathrm{JSR}_{l}$ can be calculated by subtracting the output of the NF $y(n)$ from the received signal, $r(n)$, and is given by:

$$
\mathrm{JSR}_{l \mathrm{~dB}}=10 \log _{10}\left\{|r[n]-y[n]|^{2}\right\}
$$

The transfer function of the ANF $H_{N}(z)$ [20] is given by:

$$
H_{N}(z)=\frac{N_{N}(z)}{D_{N}(Z)}=\frac{1+K_{0 l}\left(1+K_{N}\right) Z^{-1}+K_{N} Z^{-2}}{1+K_{0 l}\left(1+\beta K_{N}\right) Z^{-1}+\beta K_{N} Z^{-2}}
$$

where $k_{N}$ is the depth of the NF and is a function of the estimated $\mathrm{JSR}_{r} \beta$ is the pole radius that controls the bandwidth of the NF. Note that $N=l$. While many implementation schemes can obtain $H_{N}(z)$, this work, however, implements $H_{N}(z)$ by cascading the lattice IIR NF model [20], as shown in Figure 5. If $k_{N}$ is set equal to $1\left(k_{N}=1\right)$, the result of $H_{N}(z)$ will be the same as that 


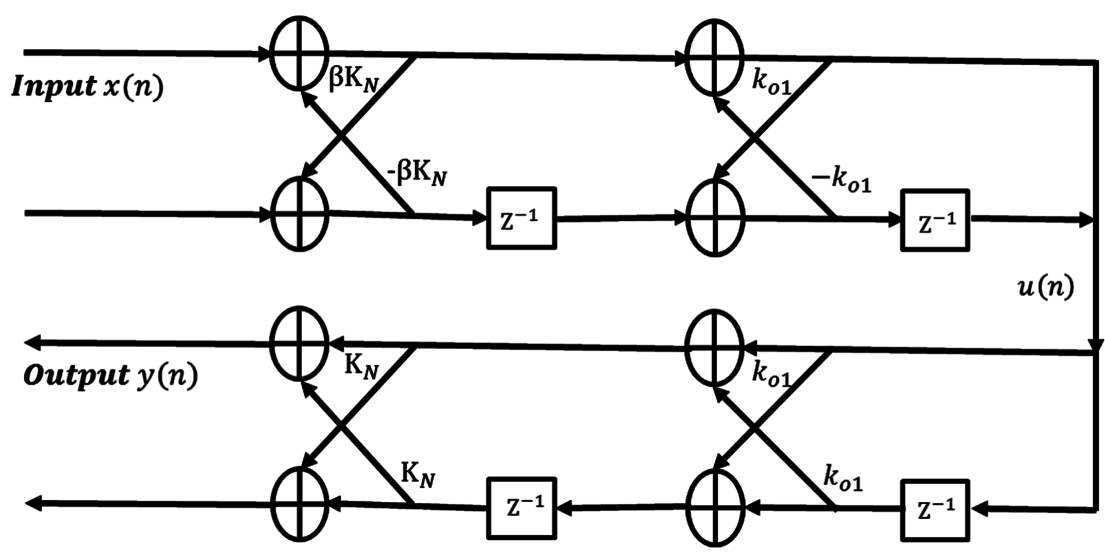

Figure 5. Lattice IIR NF structure.

of $H_{l}(z)$, which has zero on the unit circle, resulting in an infinite depth of the notch that completely removes the interference [20]. As stated previously, with the NF's infinite depth, the interference is removed completely, but nonetheless creates self-noise at the NF, resulting from the exclusion of some useful signals. Hence, the depth of the notch $k_{N}$ should thus be adjusted with the interference power to reduce the distortion of the signal. Therefore, the same structure can be used for ANF $H_{l}(z)$ and ANF $H_{N}(z)$, and the notch of Equation (17) can be placed on the IF by copying the $k_{o l}$ of ANF $H_{l}(z)$ that is tuned to IF by Equation (14) at any stage. These parameters, $\mathrm{JSR}_{b} k_{o l}$ and $k_{N}$, are needed to design the IIR notch filter $H_{N}(z)$ to maximize the output SNR at $H_{N}(z)$.

The IIR NF $H_{N}(z)$ output can be defined as:

$$
y(n)=H_{N}(z) r(n) \triangleq S_{o}(n)+w_{o}(n)+J_{o}(n)
$$

where $S_{o}(n), w_{o}(n)$ and $J_{o}(n)$ are the output components of the desired QPSK modulated signal, the white Gaussian noise, and the MCWI.

\section{Optimal Depth of the Notch that Maximizes SNR out}

This section described how the notch's optimal depth maximizes the SNR output of the IIR NF as a function of its parameters [20] [31] [32]. $\mathrm{SNR}_{\text {out }}$ can be expressed as:

$$
\mathrm{SNR}_{\text {out }}=\frac{E\left[S^{2}(n)\right]}{E\left[(y(n)-S(n))^{2}\right]}
$$

where $y(n)$ is the output of the ANF. Equation (20) describes $\mathrm{SNR}_{\text {out }}$ in terms of the filter parameters as given [20]:

$$
\mathrm{SNR}_{\text {out }}=\frac{1}{\left(1+\sigma^{2}\right)\left(\frac{\left(1+k_{N}^{2}-2 \beta k_{N}^{2}\right)}{1-\beta^{2} k_{N}^{2}}\right)+\operatorname{JSR}_{l}\left(\frac{\left(1-k_{N}\right)^{2}}{\left(1-\beta k_{N}\right)^{2}}\right)-1}
$$

where $\sigma^{2}$ is the variance of AWGN, and $\mathrm{JSR}_{l}=\frac{A_{i}^{2}}{2}$.

Let us assume that the first part of the Equation (20) denominator is $D$, called 
self-noise, as shown in Equation (21)

$$
D=\left(1+\sigma^{2}\right)\left(\frac{1+k_{N}^{2}-2 \beta k_{N}^{2}}{1-\beta^{2} k_{N}^{2}}\right)
$$

From Equation (20), the SNR output is affected by $\mathrm{JSR}_{l}$ and $D$ in Equation (21). $D$ shows a large value since $k_{N}$ increases as the notch becomes deeper, whereas $\mathrm{JSR}_{l}$ effect indicated by the second part of Equation (20). To maximize $\mathrm{SNR}_{\text {out }}$, the optimal value of $k_{N}$ needs to be found in Equation (20). The denominator in Equation (20) can be rewritten after some modification as a function of $k_{N}$ [20]:

$$
f\left(k_{N}\right)=\left(\frac{1+k_{N}^{2}-2 \beta k_{N}^{2}}{1-\beta^{2} k_{N}^{2}}\right)+G_{l}\left(\frac{\left(1-k_{N}\right)^{2}}{\left(1-\beta k_{N}\right)^{2}}\right)-\frac{1}{1+\sigma^{2}}
$$

where $G_{l}=\frac{\mathrm{JSR}_{l}}{1+\sigma^{2}}$.

To find the optimal $k_{N}$, we differentiate $f\left(k_{N}\right)$ and solve $f^{\prime}\left(k_{N}\right)=0$ for all possible roots of $k_{N}$, as shown in Equation (23):

$$
\begin{aligned}
f^{\prime}\left(k_{N}\right)= & \beta^{2} G_{l} k_{N}^{3}+\left[2 \beta G-\beta^{2} G_{l}+\beta^{2}-\beta\right] k_{N}^{2} \\
& +\left[1-\beta+G_{l}-2 \beta G\right] k_{N}-G_{l} \\
= & 0
\end{aligned}
$$

Equation (23) has at least one real root in the [0 1] range that gives the optimal $k_{N}$ as a function of $\mathrm{JSR}_{r}$. Figure 6 describes the flow chart for the system model. After a signal is received, the ANF $H_{l}(z)$ estimates $\mathrm{JSR}_{l}$ and the frequency of the interference $\left(\omega_{o l}\right)$ using Equation (16) and Equation (15), respectively. Next, and based on the estimation of $\mathrm{JSR}_{l}$ and $\hat{f}_{N}(n)$, the estimated frequency $\hat{f}_{N}(n)$ is used to place the notch by calculating $k_{o l}=-\cos \left(2 \pi \hat{f}_{N}\right)$, and then using Equation (23) to calculate the optimal value of $k_{N}$ to maximize $\mathrm{SNR}_{\text {out }}$. Then, these parameters are used to design the IIR notch filter $H_{N}(z)$ as described by Equation (17).

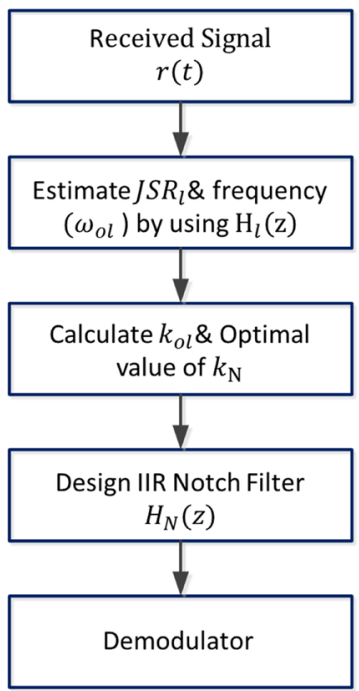

Figure 6. Flow chart of the system model. 


\section{Simulation and Discussion of Results}

The performance of the proposed low-complexity anti-jamming receiver system model of a MANF with MCWI is demonstrated in this section. The system performance is investigated in terms of the BER and the $\mathrm{SNR}_{\text {out }}$ of the IIR NF for varying JSR and SOI power $\left(E_{b} / N_{o}\right)$. In this work, for the $i^{\text {th }}$ jamming signal, the number of CWIs is considered, and thus, $m=2$ with center frequencies $\left(f_{1}=0.03\right.$ and $\left.f_{2}=0.08\right)$ in Equation (2) and with equal magnitude in the input. However, frequencies could be any value in the [0 - 1] range. The simulation was run for $1 \mathrm{e} 7$ data bits for the MCWI. $\beta$ is chosen to be 0.98 to give more satisfactory results for various situations. As $\beta$ is chosen, there is a trade-off between some properties of ANF $H_{l}(z)$ and ANF $H_{N}(z)$. A large $\beta$ value gave more accurate $\mathrm{JSR}_{l}$ and frequency estimates, but slower convergence and tracking [21] in ANF $H_{l}(z)$. However, for ANF $H_{N}(z)$, a large $\beta$ gave a larger NB noise at the output, but a smaller broadband noise [20]. The BER is measured as JSR changes.

The estimated optimal value of $k_{N}$ vs. JSR is obtained by calculating the roots as JSR is changed, with a different parameter of $\beta$ and $E_{b} / N_{o}$, as shown in Figure 7. As a result, $k_{N}$ approaches 1 as $\mathrm{JSR}_{l}$ increases to a large value. Also, this approach depends on the parameters of $\left(E_{b} / N_{o}\right)$ and $\beta$. Therefore, as the parameter of $E_{b} / N_{o}$ decrease, JSR increases as well, as $\beta \rightarrow 1$.

Figure 8, part (a), describes the frequency response of the lattice IIR notch filter for various frequencies and a fixed notch width " $\beta=0.98$ ”, where part (b) describes the frequency response of the lattice IIR notch filter for various values of $\beta$. As $\beta \rightarrow 1$, the bandwidth of the notch is decreased as well as the depth of the magnitude responses of the notch, but in some cases, the depth of the notch is infinity.

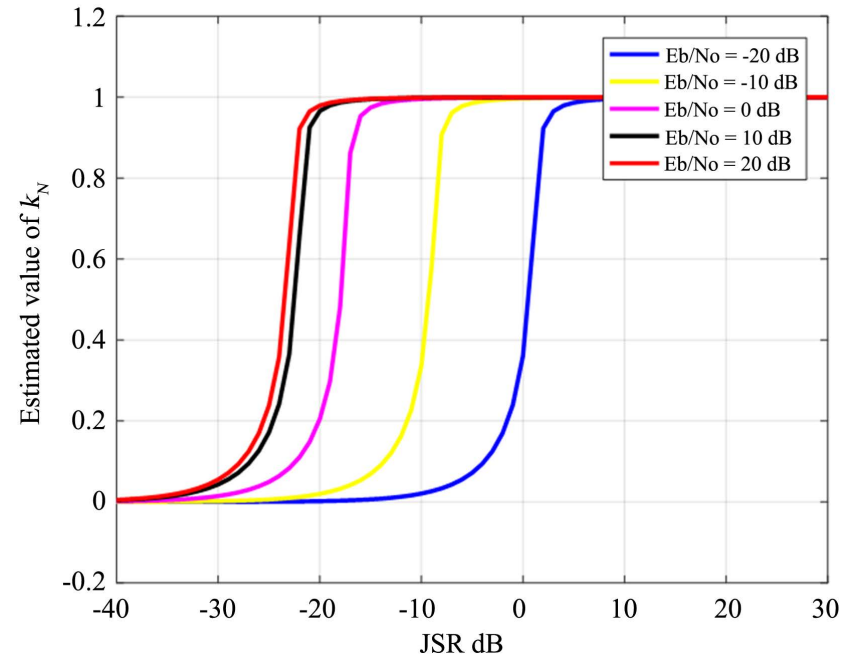

(a)

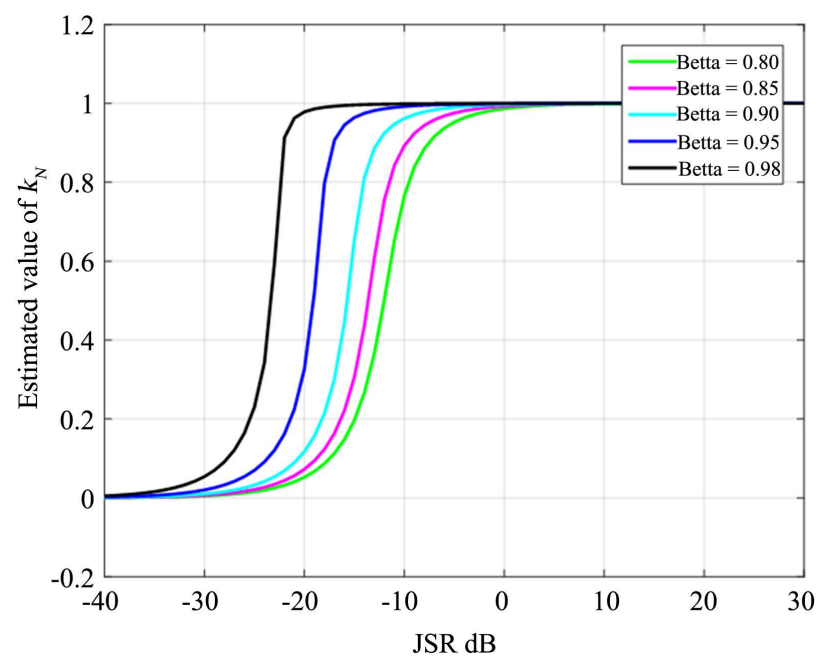

(b)

Figure 7. The estimation optimal $k_{N}$ vs. JSR: (a) with different $E_{b} / N_{o}$ parameters and $\beta=0.98$, (b) with different $\beta$ parameters and $E_{b} / N_{o}=20 \mathrm{~dB}$. 


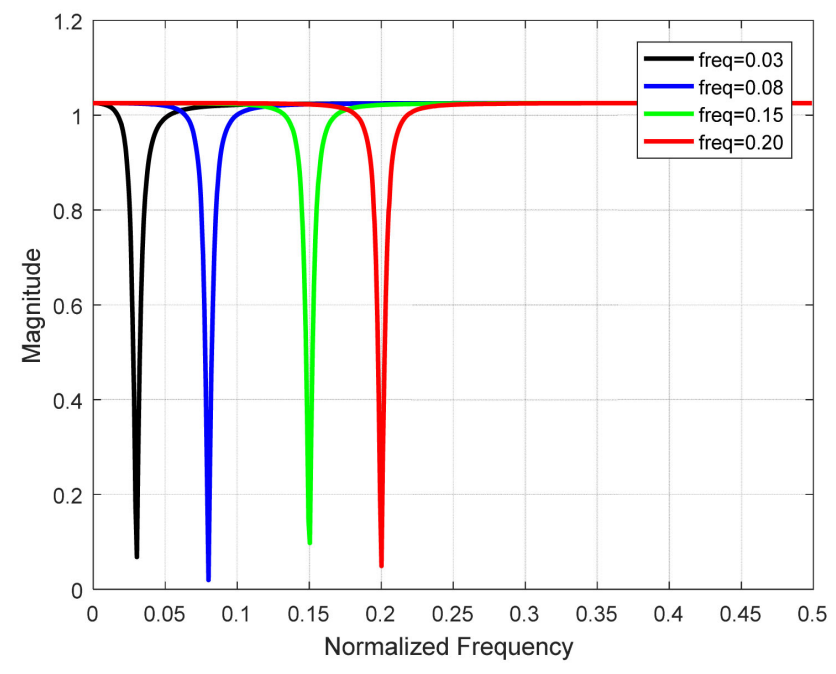

(a)

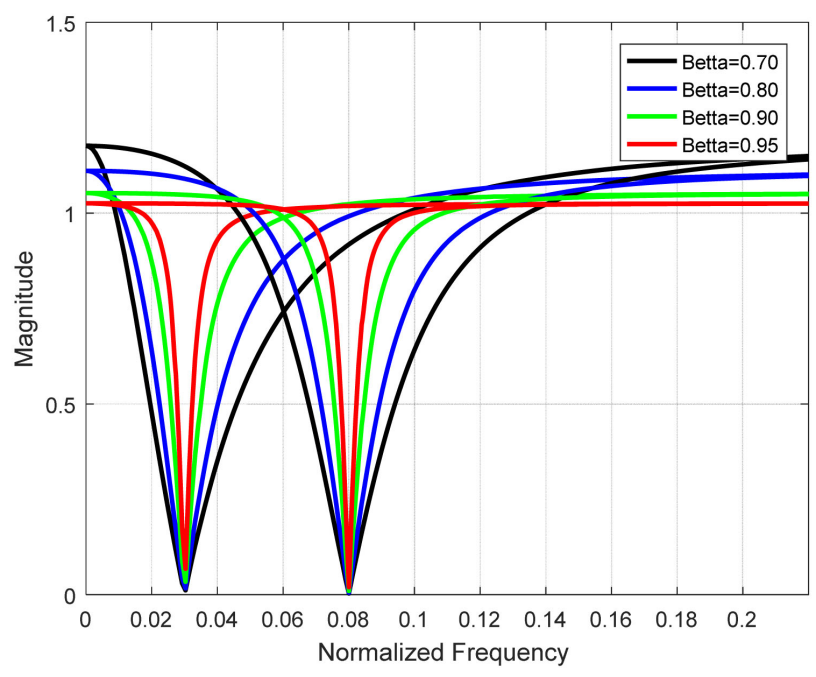

(b)

Figure 8. The frequency response of ANF: (a) with different freq parameters and $\beta=0.98$, (b) with different Betta $(\beta)$ parameters.

Figure 9 and Figure 10 show that the notch depth depends on the estimated JSR and interference frequency. Therefore, as JSR $\rightarrow$ increases, the notch magnitude response depth decrease, as does the notch bandwidth. On the other hand, the notch magnitude response depth depends on the power of the CWI; the higher power of the CWI, the deeper the notch magnitude response.

Figure 11 and Figure 12 depict the BER's performance as $E_{b} / N_{o}$ changes. The simulations were run with different values of $E_{b} / N_{o}$ and with a fixed value of JSR. The results show that the simulation with "the proposed IIR NF" filter achieved a better BER in the presence of interference, especially for a low JSR and a large $\beta$. Figure 13 and Figure 14 show the performance of the BER vs. $E_{b} / N_{o}$ in the presence of CWI and MCWI, with fixed values of JSR and different $\beta$ parameters. The results show that the filter can be able to mitigate MCWI and achieve a better BER in the presence of CWI and MCWI, with different $\beta$ s.

Figure 15 and Figure 16 show the BER's performance as JSR changes. Simulations were run with different values of JSR and a fixed $E_{b} / N_{o}$ value, with filtering and without filtering. Therefore, the results show a comparison between the simulations with filtering and without filtering. Hence, the proposed ANF algorithm with filtering provides a better result than that without filtering. Figure 17 shows a comparison between the proposed ANF algorithm and a full suppression $\left(k_{N}=1\right)$. It can be seen that the proposed algorithm effectively controls the notch depth and obtains better results than in the case of full suppression for a low JSR, and mostly the same for a high JSR.

The performance of the SNR vs. JSR is shown in Figure 18 and Figure 19. The proposed IIR NF algorithm effectively controls the notch depth, to be deeper for high JSR in each stage. The results show that full suppression achieves better results than does the case without a notch filter for a high JSR. Hence, full suppression is better for a high JSR, and without a notch filter is better for a low 


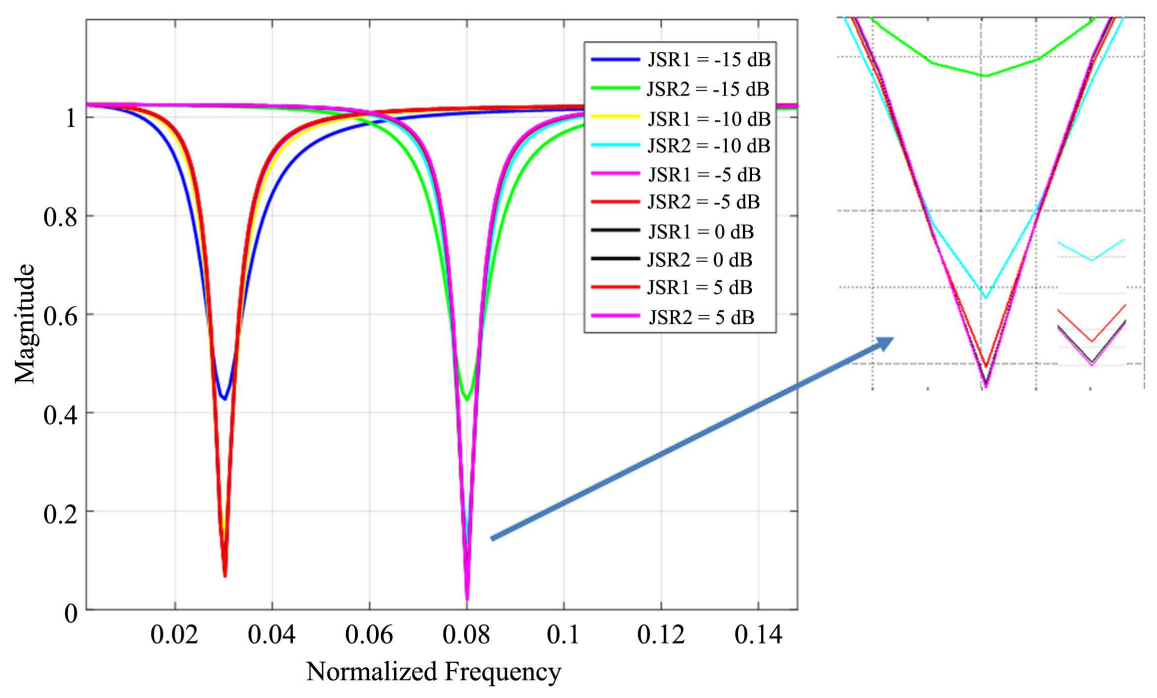

Figure 9. Notch depth vs. JSR.

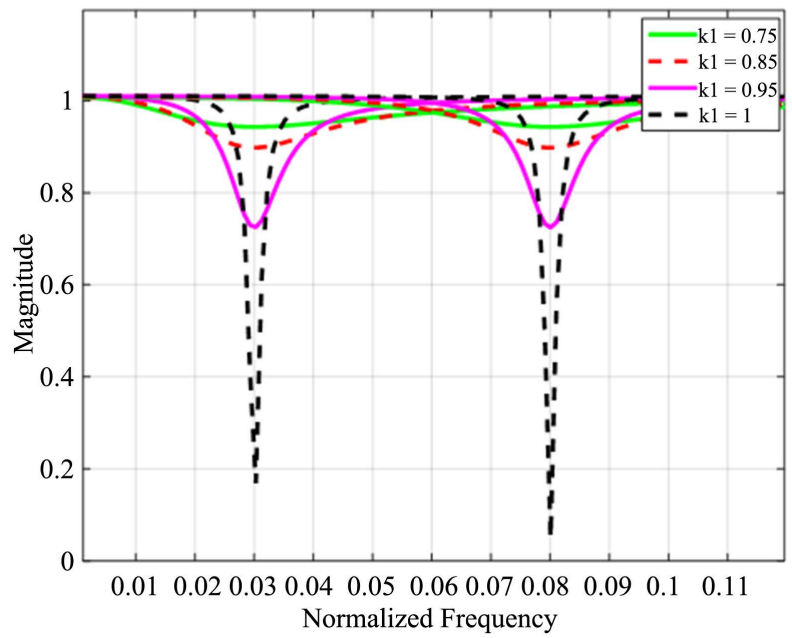

Figure 10. Notch depth vs. $k_{N}$.

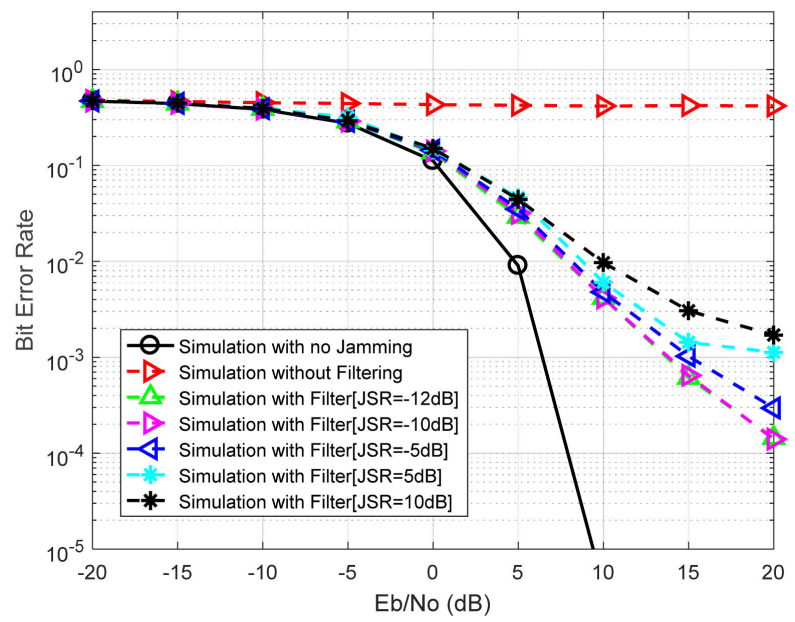

Figure 11. BER vs. $\frac{E_{b}}{N_{o}}$ with $\beta=0.98$ in presence of JSR. 


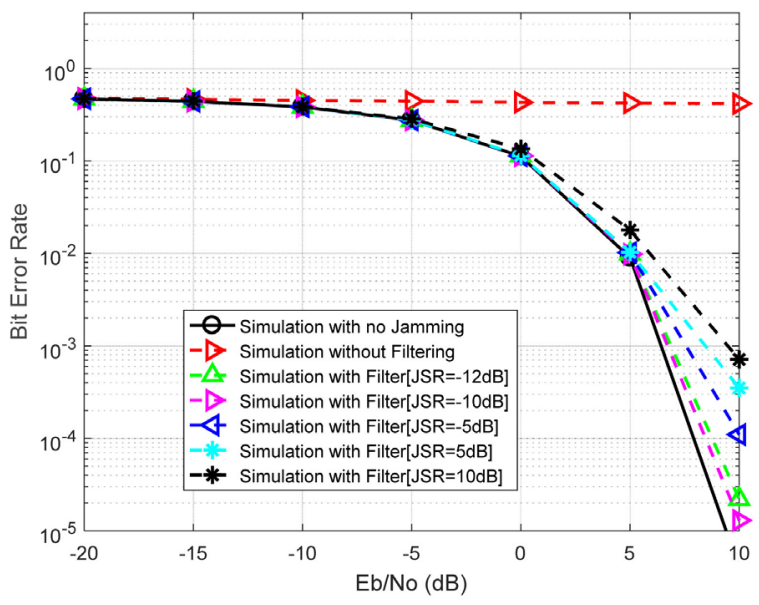

Figure 12. BER vs. $\frac{E_{b}}{N_{o}}$ with $\beta=0.999$ in presence of JSR.

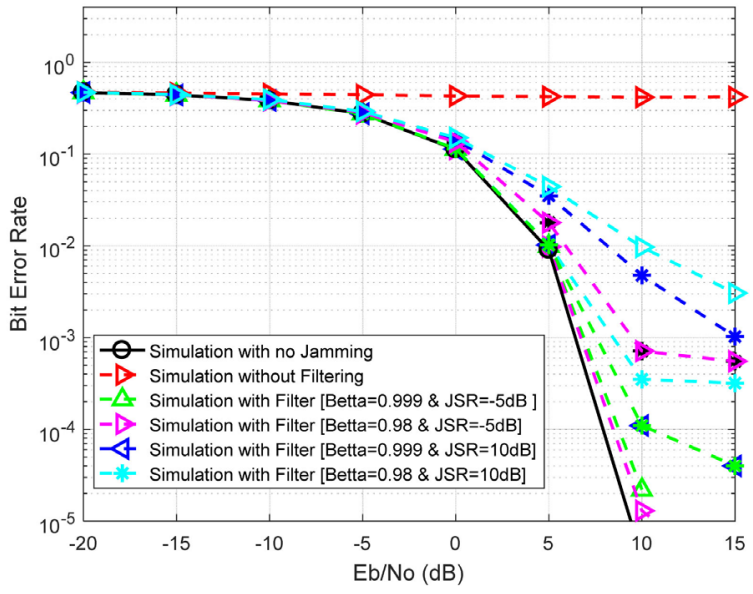

Figure 13. BER vs. $\frac{E_{b}}{N_{o}}$ with different $\beta$ and JSR parameters.

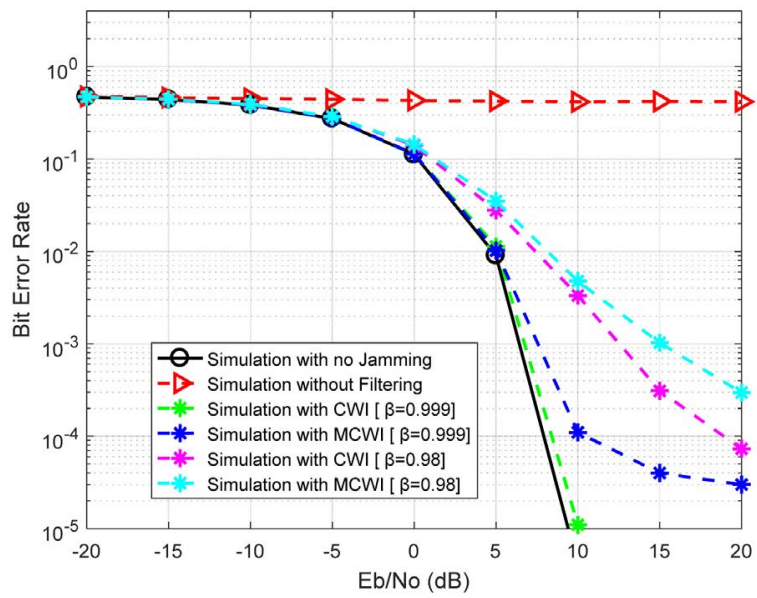

Figure 14. BER vs. $\frac{E_{b}}{N_{o}}$ with $\beta=[0.98,0.999]$ in presence of CWI and MCWI with $J S R=-5 \mathrm{~dB}$. 


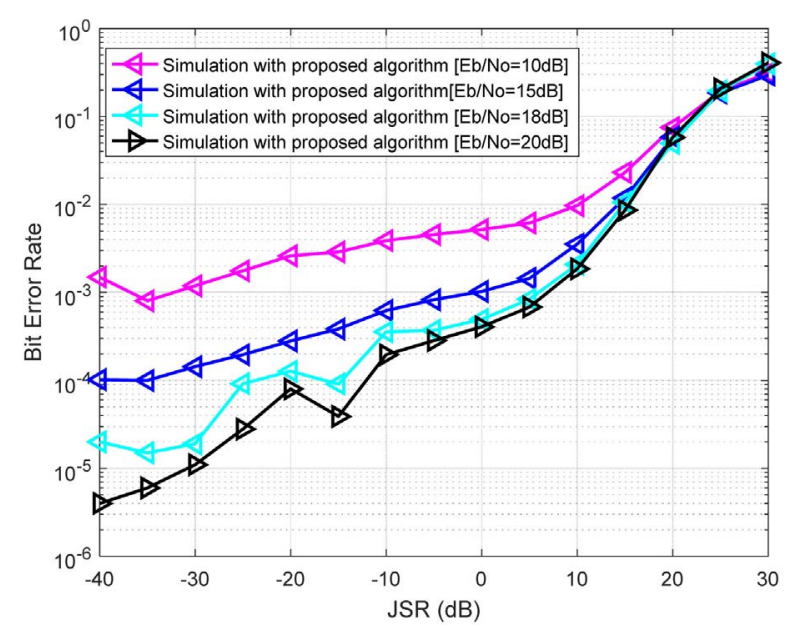

Figure 15. BER vs. JSR with $\beta=0.98$.

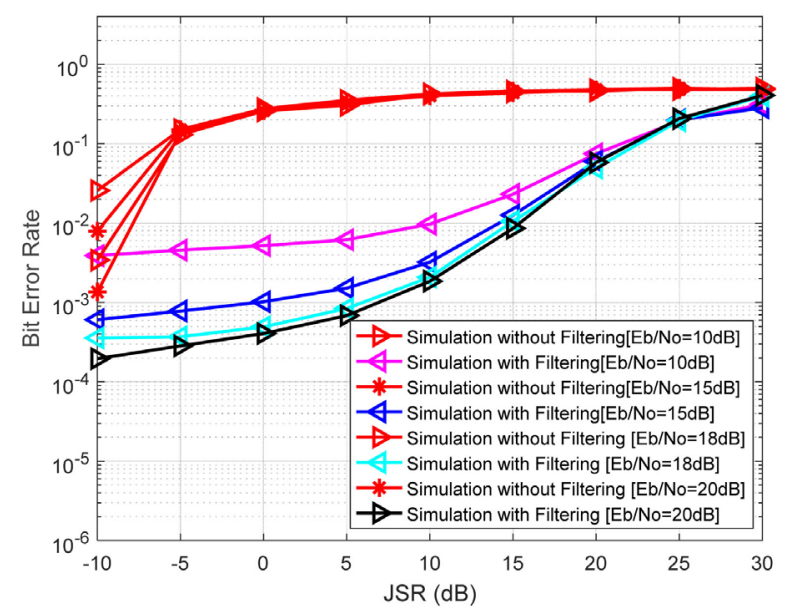

Figure 16. BER vs. JSR with $\beta=0.98, \frac{E_{b}}{N_{o}}[10,15,18,20 \mathrm{~dB}]$.

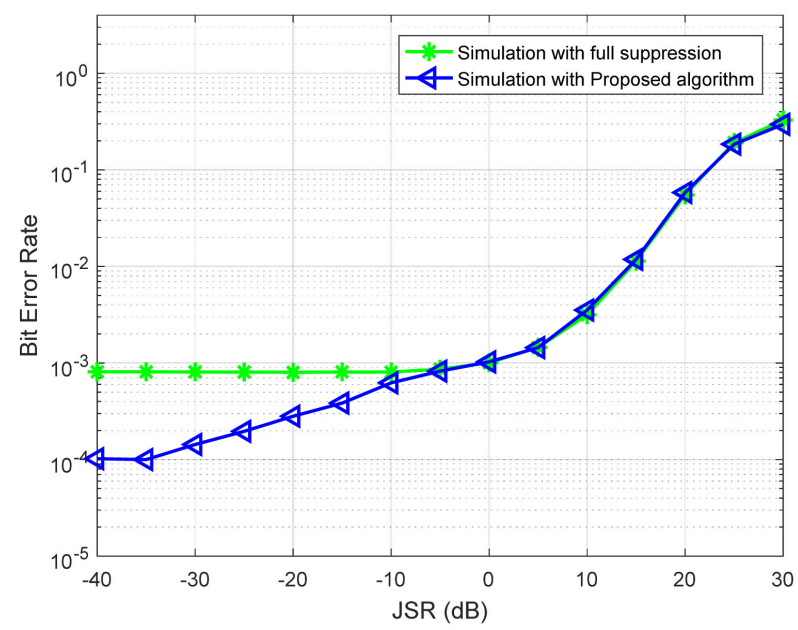

(a)

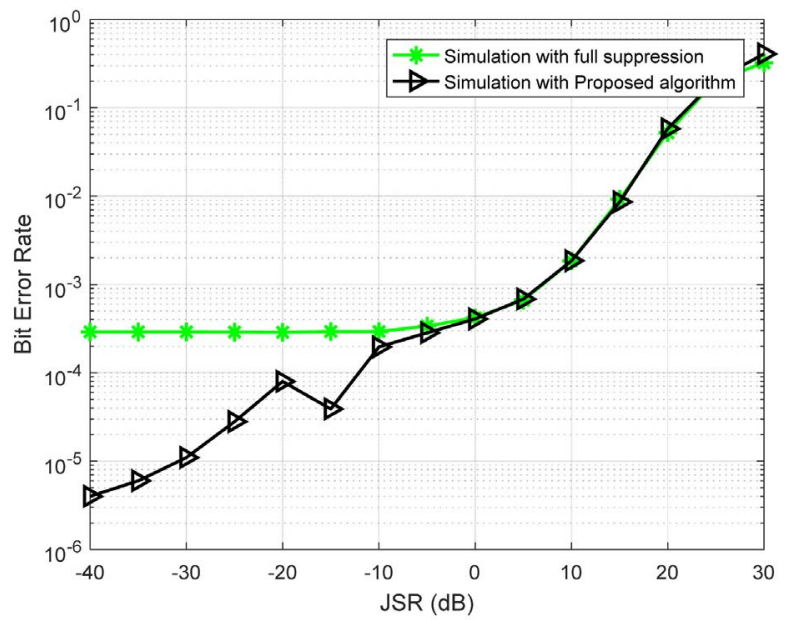

(b)

Figure 17. BER vs. JSR [ $\beta=0.98$, full suppression $\left.\left(k_{N}=1\right)\right]$; (a) $\frac{E_{b}}{N_{o}}=15 \mathrm{~dB}$, (b) $\frac{E_{b}}{N_{o}}=20 \mathrm{~dB}$. 


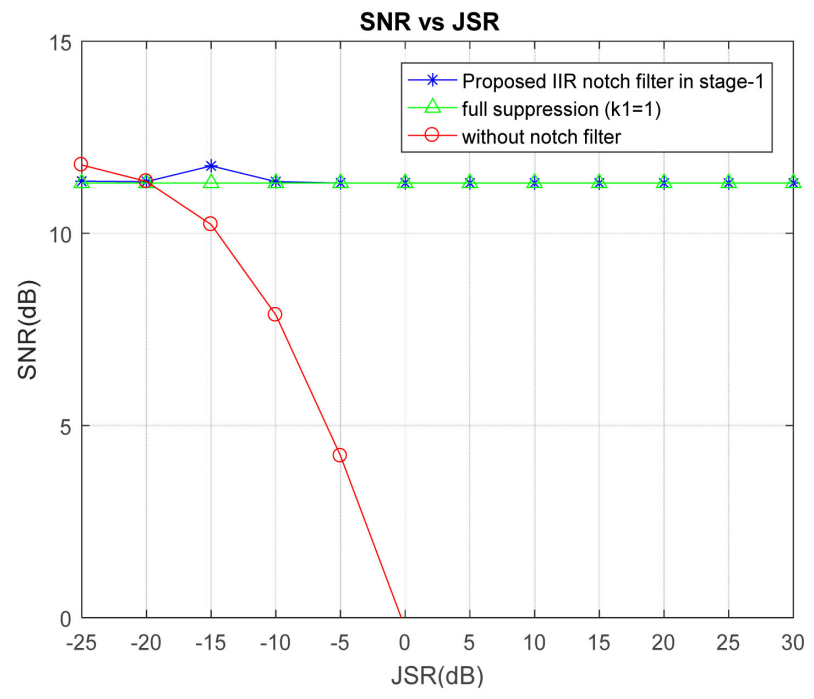

(a)

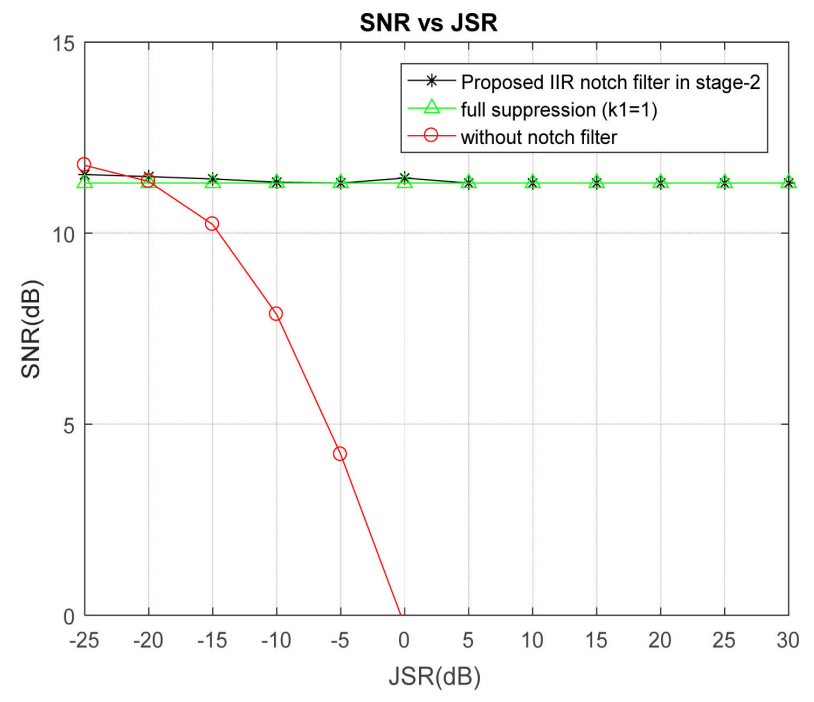

(b)

Figure 18. SNR vs. JSR with $\frac{E_{b}}{N_{o}}=15 \mathrm{~dB}$; (a) at stage-1, (b) at stage-2.

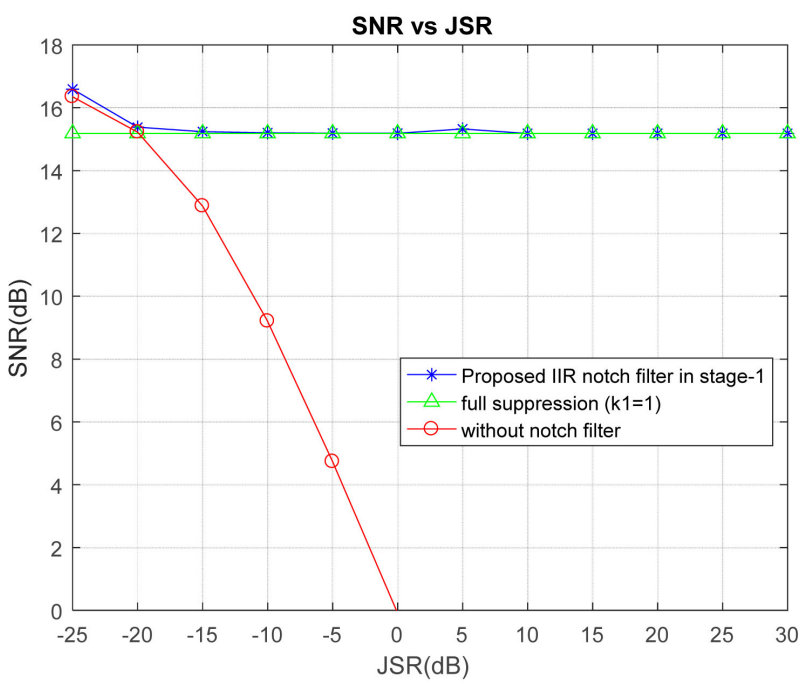

(a)

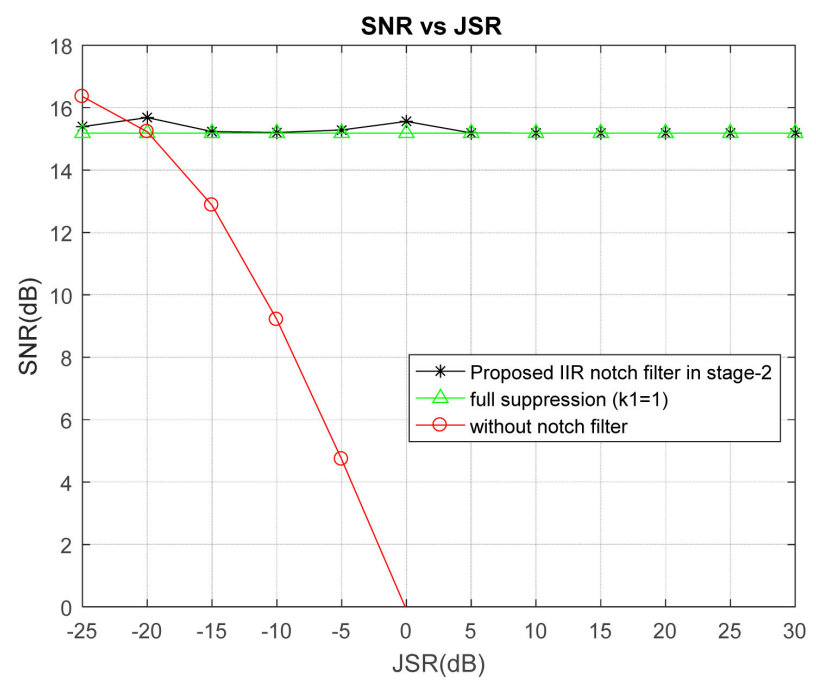

(b)

Figure 19. SNR vs. JSR with $\frac{E_{b}}{N_{o}}=20 \mathrm{~dB}$; (a) at stage-1, (b) at stage-2.

JSR. The results also show that the proposed IIR NF at any stage approaches the case of full suppression when the JSR is increasing "high" because the notch depth becomes deeper in this case. So the notch depth becomes smaller for a lower JSR value and deeper for a higher JSR value.

Figure 20 shows the received signal of the Power Spectrum (PS) before and after processing by the IIR notch filter. The results show that the proposed IIR NF algorithm is able to detect and mitigate the interference. They also show that with the adjusted depth of the IIR NF, the interference is removed according to the estimated $\mathrm{JSR}_{l}$ to avoid the reduction of the Signal of Interest (SoI). 


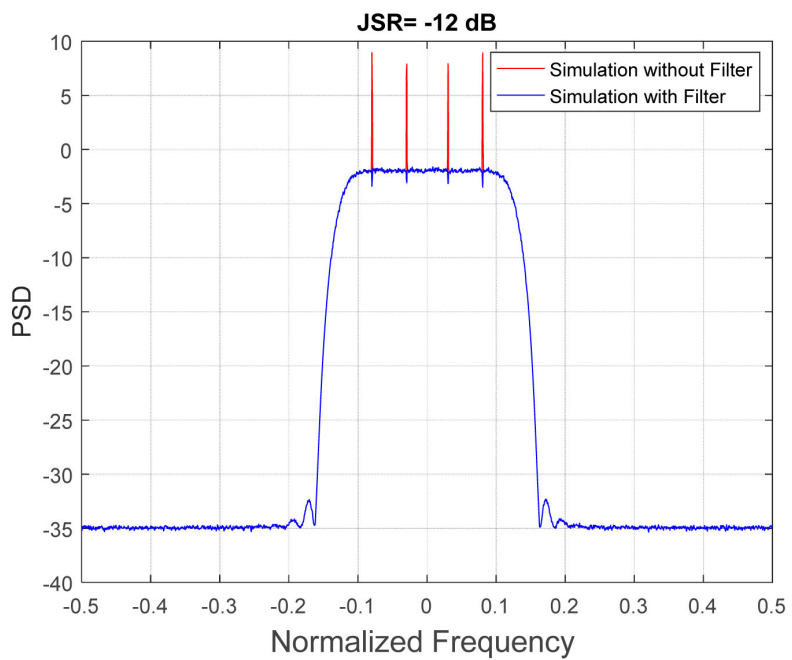

(a)

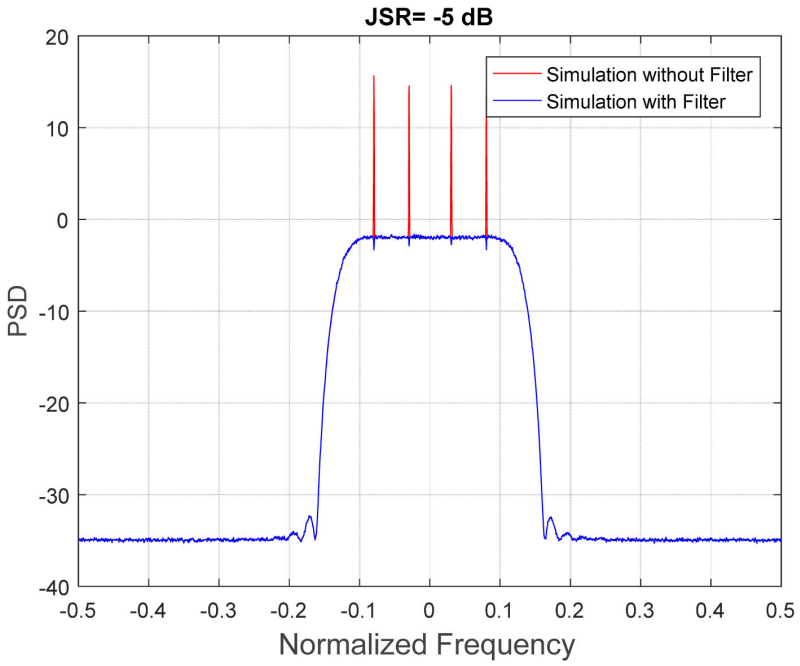

(c)

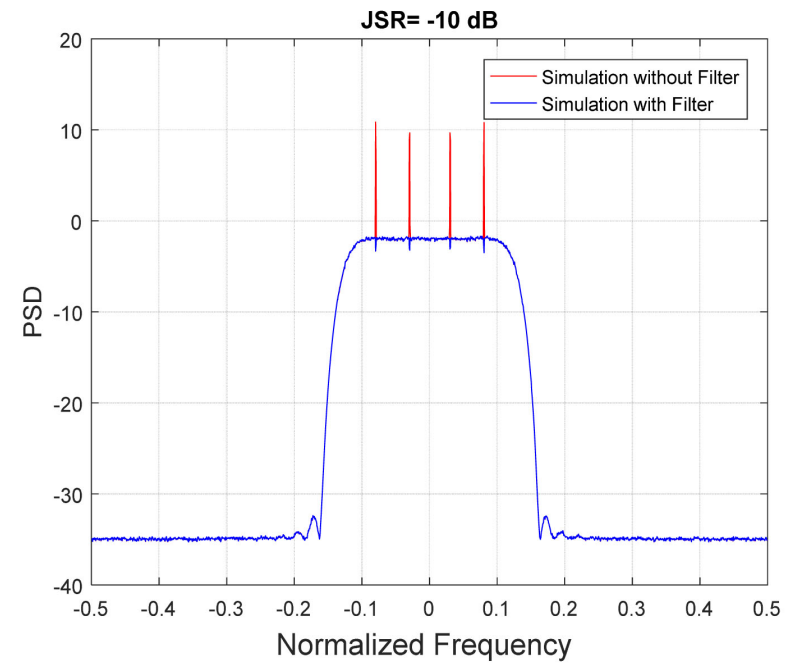

(b)

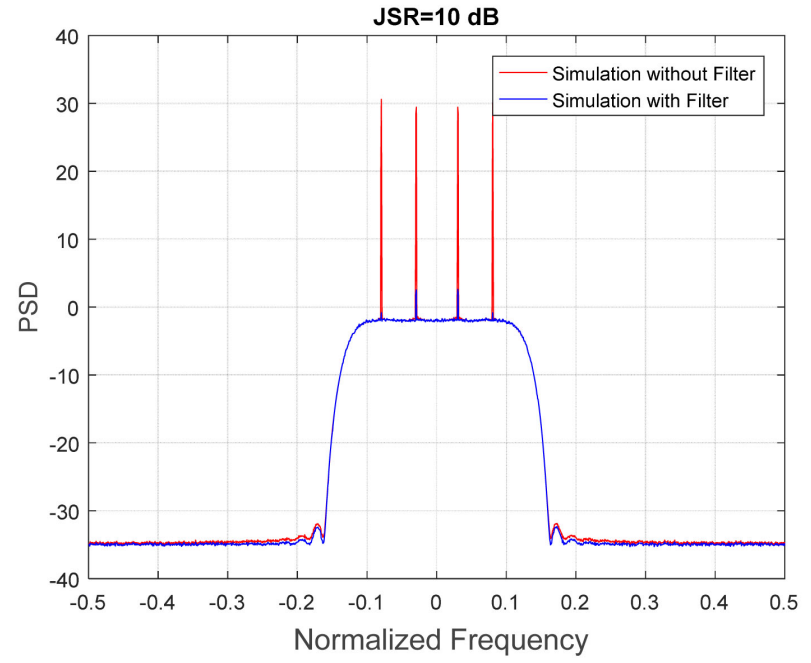

(d)

Figure 20. Input and output PSD of the notch filter with $\frac{E_{b}}{N_{o}}=20 \mathrm{~dB}, \quad \beta=0.98 ;$ (a) JSR $=-12 \mathrm{~dB}$, (b) JSR $=-10 \mathrm{~dB}$, (c) JSR $=$ $-5 \mathrm{~dB},(\mathrm{~d}) \mathrm{JSR}=10 \mathrm{~dB}$.

These results will be compared with [19]. [19] proposed a method to detect and mitigate MCWI using a simplified Welch algorithm and notch filter operating in a frequency-domain approach. The approach used a first-order IIR notch filter. Although the CWI has been removed, a portion of the useful signal has been lost, compromising overall signal integrity. Also, the main concerns with the FD approach are computational complexity and higher costs. On the other hand, the proposed method proposes a low-complexity algorithm operating in a time-domain approach using multiple ANF to detect and mitigate MCWI. The approach used the second-order IIR notch filter based on a lattice form structure. The IIR notch depth is adjusted based on interference power estimation. Table 1 shows a comparison between the algorithm proposed in [19] with our proposed algorithm. 
Table 1. Comparison between the algorithm proposed in [19] with our proposed algorithm.

\begin{tabular}{|c|c|c|}
\hline No. & Ref. [19] & Proposed algorithm \\
\hline 1 & They used QPSK signal & They used QPSK signal \\
\hline 2 & $\begin{array}{l}\text { They proposed a method to detect } \\
\text { and mitigate MCWI using a simplified } \\
\text { Welch algorithm and notch filter }\end{array}$ & $\begin{array}{l}\text { We proposed a low-complexity algorithm } \\
\text { to detect and mitigate MCWI using a } \\
\text { multiple adaptive notch filter based on the } \\
\text { lattice form structure }\end{array}$ \\
\hline 3 & They used a frequency-domain approach & We used a time-domain approach \\
\hline 4 & $\begin{array}{l}\text { They used a first-order } \\
\text { IIR adaptive notch filter }\end{array}$ & $\begin{array}{l}\text { we used a second-order } \\
\text { IIR adaptive notch filter }\end{array}$ \\
\hline 5 & They do not adjust the depth of the notch & $\begin{array}{l}\text { We adjusted the depth of the notch } \\
\text { based on the estimation of the JSR }\end{array}$ \\
\hline
\end{tabular}

\section{Conclusion}

This paper proposes a novel low-complexity algorithm for mitigating multi-tone continuous wave interference using multiple adaptive notch filters based on a second-order IIR lattice form structure. This structure detects, estimates, and removes the MCWI. The algorithm operates in TD, which reduces hardware costs. Adaptive frequency estimators are used to adjust the notch filter's zeros, placing them on the interference frequency. However, if zeros on the unit circle and has a notch of infinite depth $\left(k_{N}=1\right)$, and leading to the complete removal of the interference and exclusion of some desired signals This causes a reduction of the Signal of Interest (SoI) and results in a degraded QoS, a decrease in SNR, and an increase in BER. The adaptive notch filter algorithm for the given notch filter is thus proposed to adjust the notch depth and frequency. Therefore, the proposed method can effectively detect and mitigate the MCWI and adjust the notch depth for any given value of JSR, and can therefore effectively control the notch depth. It also provides better results than does a full suppression for low JSR values, and mostly the same performance for high JSR values, and provides a better BER performance. Also, the resulting $\mathrm{SNR}_{\text {out }}$ of the $\mathrm{NF}$ is maximized for lower and higher JSR values with different values of $\left(E_{b} / N_{o}\right)$ power. Therefore, this technique can be applied in a DVB-S2 receiver or any other communication and navigation receivers.

\section{Acknowledgements}

The work reported in this paper was done under the AVIO 601-Interference Mitigation in Satellite Communication Project of the LASSENA Lab, École de Technologie Supérieure (ÉTS). This research was also supported by the Natural Sciences and Engineering Research Council of Canada (NSERC), Thales, Telesat, Vigilant Global, CRIAQ, and Atem Canada.

\section{Conflicts of Interest}

The authors declare no conflicts of interest regarding the publication of this paper. 


\section{References}

[1] Lu, D., Wu, R. and Liu, H. (2013) Global Positioning System Anti-Jamming Algorithm Based on Period Repetitive CLEAN. IET Radar, Sonar \& Navigation, 7, 164-169. https://doi.org/10.1049/iet-rsn.2010.0353

[2] Lin, T., Abdizadeh, M., Broumandan, A., Wang, D., O’Keefe, K. and Lachapelle, G. (2011) Interference Suppression for High Precision Navigation Using Vector-Based GNSS Software Receivers. Proceedings of the 24th International Technical Meeting of The Satellite Division of the Institute of Navigation, Portland, 19-23 September 2011, 372-383.

[3] Glennon, E.P. and Dempster, A.G. (2011) Delayed PIC for Postcorrelation Mitigation of Continuous Wave and Multiple Access Interference in GPS Receivers. IEEE Transactions on Aerospace and Electronic Systems, 47, 2544-2557.

https://doi.org/10.1109/TAES.2011.6034650

[4] Choi, J.W. and Cho, N.I. (2001) Narrow-Band Interference Suppression in Direct Sequence Spread Spectrum Systems Using a Lattice IIR Notch Filter. 2001 IEEE International Conference on Acoustics, Speech, and Signal Processing, Salt Lake City, 7-11 May 2001, 2237-2240. https://doi.org/10.1109/ICASSP.2001.940443

[5] Barbarossa, S. and Scaglione, A. (1999) Adaptive Time-Varying Cancellation of Wideband Interferences in Spread-Spectrum Communications Based on Time-Frequency Distributions. IEEE Transactions on Signal Processing, 47, 957-965.

https://doi.org/10.1109/78.752594

[6] Amin, M.G., Wang, C. and Lindsey, A.R. (1999) Optimum Interference Excision in Spread Spectrum Communications Using Open-Loop Adaptive Filters. IEEE Transactions on Signal Processing, 47, 1966-1976.

https://doi.org/10.1109/78.771045

[7] Ma, W.J., Mao, W.-L. and Chang, F.R. (2005) Design of Adaptive All-Pass Based Notch Filter for Narrowband Anti-Jamming GPS System. 2005 International Symposium on Intelligent Signal Processing and Communication Systems, Hong Kong, 13-16 December 2005, 305-308. https://doi.org/10.1109/ISPACS.2005.1595407

[8] Lv, Q. and Qin, H. (2018) A Novel Algorithm for Adaptive Notch Filter to Detect and Mitigate the CWI for GNSS Receivers. 2018 IEEE 3rd International Conference on Signal and Image Processing, Shenzhen, 13-15 July 2018, 444-451. https://doi.org/10.1109/SIPROCESS.2018.8600453

[9] Chien, Y.R. (2013) Design of GPS Anti-Jamming Systems Using Adaptive Notch Filters. IEEE Systems Journal, 9, 451-460.

https://doi.org/10.1109/JSYST.2013.2283753

[10] Lv, Q. and Qin, H. (2020) General Method to Mitigate the Continuous Wave Interference and Narrowband Interference for GNSS Receivers. IET Radar, Sonar \& Navigation, 14, 1430-1435. https://doi.org/10.1049/iet-rsn.2020.0115

[11] Ouyang, X. and Amin, M.G. (2001) Short-Time Fourier Transform Receiver for Nonstationary Interference Excision in Direct Sequence Spread Spectrum Communications. IEEE Transactions on Signal Processing, 49, 851-863. https://doi.org/10.1109/78.912929

[12] Zhang, Y., Amin, M.G. and Lindsey, A.R. (2001) Anti-Jamming GPS Receivers Based on Bilinear Signal Distributions. 2001 MILCOM Proceedings Communications for Network-Centric Operations. Creating the Information Force, McLean, 28-31 October 2001, 1070-1074. https://doi.org/10.1109/MILCOM.2001.986006

[13] Jiang, F.W., Xiao, Z., Yi, K.C. and Sun, Y.J. (2008) Adaptive Two-Stage Filter Bank 
Based Narrowband Interference Suppression in DSSS Systems. 2008 9th International Conference on Signal Processing, Beijing, 26-29 October 2008, 88-91. https://doi.org/10.1109/ICOSP.2008.4697075

[14] Dovis, F. and Musumeci, L. (2011) Use of Wavelet Transforms for Interference Mitigation. 2011 International Conference on Localization and GNSS, Tampere, 29-30 June 2011, 116-121. https://doi.org/10.1109/ICL-GNSS.2011.5955275

[15] Landry, R.J., Mouyon, P. and Lekaïm, D. (1998) Interference Mitigation in Spread Spectrum Systems by Wavelet Coefficients Thresholding. European Transactions on Telecommunications, 9, 191-202. https://doi.org/10.1002/ett.4460090209

[16] Capozza, P.T., Holland, B.J., Hopkinson, T.M. and Landrau, R.L. (2000) A Single-Chip Narrow-Band Frequency-Domain Excisor for a Global Positioning System (GPS) Receiver. IEEE Journal of Solid-State Circuits, 35, 401-411. https://doi.org/10.1109/4.826823

[17] Lotz, T. (2008) Adaptive Analog-to-Digital Conversion and Pre-Correlation Interference Mitigation Techniques in a GNSS Receiver. Diploma Thesis, Technical University of Kaiserslautern, Kaiserslautern.

[18] Balaei, A.T. and Dempster, A.G. (2009) A Statistical Inference Technique for GPS Interference Detection. IEEE Transactions on Aerospace and Electronic Systems, 45, 1499-1511. https://doi.org/10.1109/TAES.2009.5310313

[19] Same, M.H., Gleeton, G., Gandubert, G., Ivanov, P. and Landry, R. (2021) Multiple Narrowband Interferences Characterization, Detection and Mitigation Using Simplified Welch Algorithm and Notch Filtering. Applied Sciences, 11, Article No。 1331. https://doi.org/10.3390/app11031331

[20] Choi, J.W. and Cho, N.I. (2002) Suppression of Narrow-Band Interference in DSSpread Spectrum Systems Using Adaptive IIR Notch Filter. Signal Processing, 82, 2003-2013. https://doi.org/10.1016/S0165-1684(02)00385-7

[21] Cho, N.I. and Lee, S.U. (1993) On the Adaptive Lattice Notch Filter for the Detection of Sinusoids. IEEE Transactions on Circuits and Systems II: Analog and Digital Signal Processing, 40, 405-416. https://doi.org/10.1109/82.238368

[22] Cho, N.I., Choi, C.-H. and Lee, S.U. (1989) Adaptive Line Enhancement by Using an IIR Lattice Notch Filter. IEEE Transactions on Acoustics, Speech, and Signal Processing, 37, 585-589. https://doi.org/10.1109/29.17543

[23] Borio, D., Camoriano, L. and Presti, L.L. (2008) Two-Pole and Multi-Pole Notch Filters: A Computationally Effective Solution for GNSS Interference Detection and Mitigation. IEEE Systems Journal, 2, 38-47. https://doi.org/10.1109/JSYST.2007.914780

[24] Vijayan, R. and Poor, H.V. (1990) Nonlinear Techniques for Interference Suppression in Spread-Spectrum Systems. IEEE Transactions on Communications, 38, 1060-1065. https://doi.org/10.1109/26.57504

[25] Wang, K.-J. and Yao, Y. (1999) New Nonlinear Algorithms for Narrowband Interference Suppression in CDMA Spread-Spectrum Systems. IEEE Journal on Selected Areas in Communications, 17, 2148-2153. https://doi.org/10.1109/49.814831

[26] Wu, W.-R. and Yu, F.-F. (1996) New Nonlinear Algorithms for Estimating and Suppressing Narrowband Interference in DS Spread Spectrum Systems. IEEE Transactions on Communications, 44, 508-515. https://doi.org/10.1109/26.489097

[27] Ferdjallah, M. and Barr, R.E. (1994) Adaptive Digital Notch Filter Design on the Unit Circle for the Removal of Powerline Noise from Biomedical Signals. IEEE Transactions on Biomedical Engineering, 41, 529-536. 
https://doi.org/10.1109/10.293240

[28] Borio, D., Camoriano, L., Savasta, S. and Presti, L.L. (2008) Time-Frequency Excision for GNSS Applications. IEEE Systems Journal, 2, 27-37.

https://doi.org/10.1109/JSYST.2007.914914

[29] El Gebali, A. and Landry Jr., R. (2020) Mitigation of Continuous Wave Narrow-Band Interference in QPSK Demodulation Using Adaptive IIR Notch Filter. American Journal of Signal Processing, Scientific \& Academic Publishing, 10, 10-18.

[30] Punchalard, R. (2002) On Adaptive IIR Lattice Notch Filter Using a Robust Variable Step-Size for the Detection of Sinusoid. The 2002 8th International Conference on Communication Systems, Singapore, 28 November 2002, 800-804.

https://doi.org/10.1109/ICCS.2002.1183240

[31] Batra, A. and Zeidler, J.R. (2008) Narrowband Interference Mitigation in OFDM Systems. 2008 IEEE Military Communications Conference, San Diego, 16-19 November 2008, 1-7. https://doi.org/10.1109/MILCOM.2008.4753296

[32] Ahn, J., Kim, J. and Kim, K. (2009) Adaptive Interference Suppression Methods Using Transform Domain Approach in an On-Board Filter Bank for Satellite Communications. TENCON 2009-2009 IEEE Region 10 Conference, Singapore, 23-26 January 2009, 1-5. https://doi.org/10.1109/TENCON.2009.5395990 